\title{
RECONCILIAÇÃO DE DADOS PARA MEDIÇÃO DE GÁS NATURAL EM MALHA DE DUTOS
}

Ademário Lopes Ferreira dos Santos 1; Eduardo Gertrudes 2; Fernando Luiz Pellegrini Pessoa ${ }^{3}$ Ewerton Emmanuel da Silva Calixto ${ }^{4}$

${ }^{1}$ Companhia de Gás da Bahia (BAHIAGAS)/Centro Universitário SENAI CIMATEC; Salvador/Bahia; ademariolopes@hotmail.com ademariosantos@bahiagas.com.br.

${ }^{2}$ Universidade Federal do Rio Grande do Norte; Natal/RN;

${ }^{3}$ Centro Universitário SENAI CIMATEC; Salvador/Bahia;

${ }^{4}$ Centro Universitário SENAI CIMATEC; Salvador/Bahia

Resumo: $O$ impacto econômico associado à medição de gás natural exige uma otimização do controle do balanço volumétrico no sistema de entrega. O objetivo deste trabalho foi avaliar a medição de gás natural na concessionária de distribuição de gás natural para malha de dutos no estado da Bahia, através da técnica da reconciliação de dados, além de interagir a avaliação de medição com a margem de faturamento do preço de venda deste produto. Os resultados do estudo possibilitam discutir a adequação da técnica de reconciliação de dados. Dentre as conclusões deste trabalho, os resultados obtidos demonstram que a técnica de reconciliação de dados pode contribuir para identificação de falhas, assim como agregar qualidade às medições de gás natural.

Palavras-Chave: Reconciliação de Dados; Medição; Gás Natural; Faturamento.

\section{DATA RECONCILIATION FOR MEASUREMENT OF NATURAL GAS IN DUTCH MATERIAL}

Abstract: The economic impact associated with the measurement of natural gas requires an optimization of volumetric balance control in the delivery system. The objective of this work was to evaluate the measurement of natural gas in the concessionaire for the distribution of natural gas for pipelines in the state of Bahia, through the technique of data reconciliation, in addition to interacting the measurement evaluation with the price margin of the price of sale of this product. The results of the study make it possible to discuss the adequacy of the data reconciliation technique. Among the conclusions of this work, the results obtained demonstrate that the data reconciliation technique can contribute to the identification of failures, as well as to add quality to natural gas measurements.

Keywords: Data Reconciliation; Measurement; Natural gas; Revenues. 


\section{INTRODUÇÃO}

Sobre medição de gás natural, medição confiável é a forma mais adequada para se contabilizar o gás natural em conformidade à legislação vigente. Se faz necessária a atribuição de confiabilidade metrológica aos equipamentos à técnica de medição utilizada. Como se trata de um composto leve e volátil, seu transporte é vulnerável a perdas de massa do produto, o que gera prejuízos para os investidores [1,9]. A qualidade da medição de gás natural pode ser comprometida pelo uso de métodos inadequados de medição, devido ao cumprimento inadequado de normas prescritas de medição, instalação inadequada e falta de calibração de equipamentos. Ausência de turbulência, desenvolvimento do perfil de velocidades a montante do medidor, variações nas propriedades do fluido e condições do escoamento, são alguns dos fatores podem comprometer a qualidade da medição $[1,9]$.

Dados disponíveis são medidos por instrumentos e equipamentos que apresentam variabilidade, incertezas de medição e estão sujeitos a falhas. Além disto, também existem variabilidade e falhas inerentes ao próprio processo. Consequentemente, não deve ser esperado que todas as medições de uma planta respeitem o modelo matemático adotado para interpretação dos dados obtidos [7,8]. Desta forma, faz-se necessário que os dados sejam processados antes de passar por alguma análise ou processamento posterior. A reconciliação de dados é uma técnica desenvolvida para este fim ${ }^{[6,7]}$. A reconciliação de dados é uma técnica de ajuste das medições obtidas em um processo, de modo que estas atendam ao modelo matemático usado para descrever o processo. Esta técnica leva em conta a natureza estatística de cada medição e está fundamentada no fato de que as plantas industriais dispõem de instrumentação abundante $\mathrm{e}$, consequentemente, redundância de informação. A formulação do problema de reconciliação de dados é baseada na minimização de uma função objetivo sujeita a restrições definidas pela natureza do processo ${ }^{[4,6]}$.

A pesquisa em pauta trata-se de uma reconciliação de dados não linear, cuja função objetivo trata-se uma função quadrática. A função objetivo típica de reconciliação de dados para o balanço em estudo é representada pela equação ${ }^{[3]}$ :

$$
\min \sum_{i=1}^{N}\left(\frac{\sum V R_{i}-\sum V M_{i}}{\sigma_{i}^{2}}\right)^{2}
$$

Sujeito as seguintes restrições de balanços volumétrico:

$$
\sum_{i=1}^{L}\left(V R_{i}\right)_{i n j}-\sum_{m=1}^{M}\left(V R_{i}\right)_{o u t j}=0 \quad \mathrm{j}=1, \ldots \mathrm{J}
$$

Onde:

i refere-se às correntes;

$\sigma_{\mathrm{i}}=\mathrm{a}$ incerteza associada à medição;

$V R_{i}=$ vazões reconciliadas; 
$\mathrm{VM}_{\mathrm{i}}=$ vazões medidas;

in = representa as correntes de entrada;

out = correntes de saída;

$\mathrm{N}=\mathrm{n}$ ㅇ total de correntes envolvidas;

$\mathrm{L}=$ correntes de entrada em cada unidade;

$\mathrm{j}=$ refere-se a cada unidade;

$\mathrm{J}=$ quantidades de unidades.

Além das restrições de balanço (equação 2), são apontadas também outras restrições tais como: a impossibilidade de vazões negativas (restrições de não negatividade) e restrições operacionais (determinadas vazões possuem limites), geralmente este tipo de restrição é inserido com base em informações proveniente de especialistas da área [5].

O problema geral de reconciliação não linear pode ser formulado como o problema da minimização por mínimos quadrados, tal como segue:

$$
\min _{x, y}(y-x)^{T} \psi^{-1}(y-x)
$$

Sujeito as seguintes restrições:

$$
\begin{aligned}
& f(x, u)=0 \\
& g(x, u) \leq 0
\end{aligned}
$$

Onde:

$\mathrm{f}$ : vetor $m \times 1$ das restrições de igualdade;

$\mathrm{g}$ : vetor $\mathrm{q} \times 1$ das restrições de desigualdade;

$\psi$ : matriz $\mathrm{n} \times \mathrm{n}$ de covariância;

$\mathrm{u}$ : vetor $\mathrm{p} \times 1$ das variáveis não medidas;

$\mathrm{x}$ : vetor $\mathrm{n} \times 1$ das variáveis medidas;

$\mathrm{y}$ : vetor $\mathrm{n} \times 1$ dos valores medidos das variáveis $\mathrm{x}$.

\subsection{Objetivos da Pesquisa}

Avaliar a medição de gás natural de uma concessionaria de distribuição de gás natural, tomando-se como modelo um "recorte" de parte da malha medição, comparando-se as medições entre a ETC (Estação de Transferência de Custódia) e os pontos de consumo para os diversos clientes dessa concessionária, aplicando a técnica de Reconciliação de Dados, afim de verificar a relação entre consumo e fornecimento e avaliar a diferença de preço/faturamento entre as medições não 
reconciliadas e reconciliadas, tanto no balanço global, quanto no comparativo individual por cliente.

\section{METODOLOGIA}

Segue breve desenho esquemático exemplificando um fluxograma entre a ETC e distribuição de gás para clientes consumidores:

Figura 1 - Modelo de fluxograma ETC x Clientes

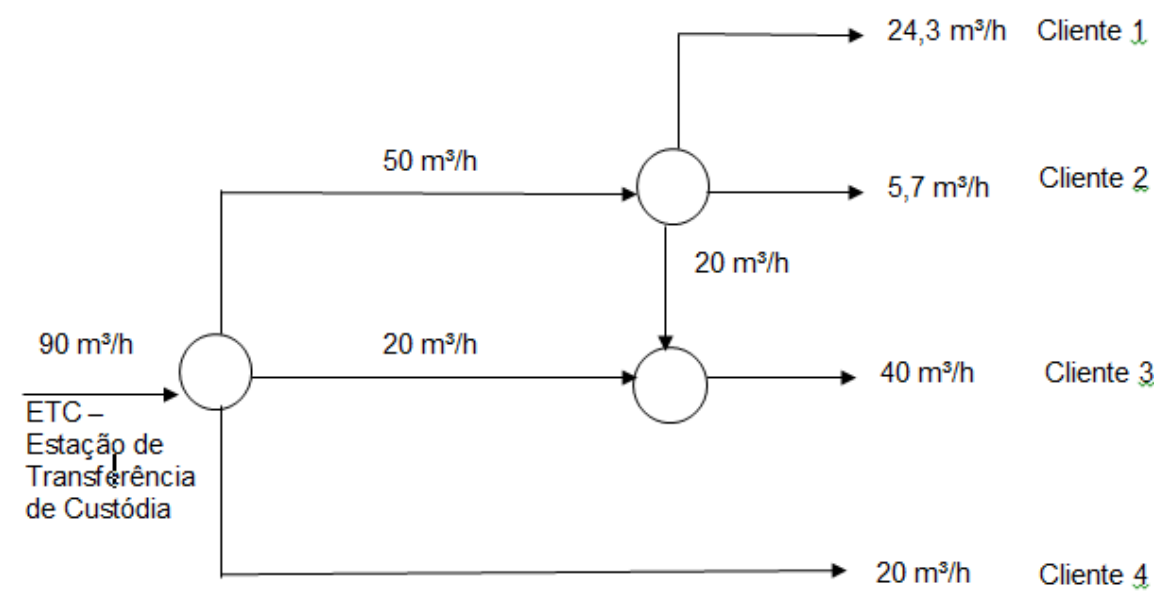

Inicialmente, foi realizada coleta das medições de gás natural, tomando-se como modelo um "recorte" de parte da malha medição, comparando-se as medições entre a ETC (Estação de Transferência de Custódia) e os pontos de consumo para os diversos clientes dessa concessionária, aplicando a técnica de Reconciliação de Dados, onde foram utilizados dados quantitativos de medições diárias.

Foram avaliados a medição de gás de sete clientes durantes cinco dias. A cada dia, foi comparado a medição de gás do ponto de saída (ETC) e os oito clientes. Teoricamente, a diferença entre as vazões da ETC e somatório dos clientes deverá ser igual a zero, mas devido aos dados disponíveis serem medidos por instrumentos e equipamentos que apresentam variabilidade, incertezas de medição e estão sujeitos a falhas, além das variabilidade e falhas inerentes ao próprio processo, fazem com que essa restrição não seja inicialmente obedecida.

Logo após, realizou-se a reconciliação de dados, além da comparação sob a ótica de faturamento (monetário) entre as diferenças das medições reconciliadas e não reconciliadas, obedecendo as restrições balanço volumétrico (diferença entre entrada e saída igual a zero, conforme equação 2) além das restrições relacionadas a não negatividades, ou seja, os valores de medições serem sempre positivos.

O procedimento para coleta de dados foi da seguinte forma: Medições diretamente dos medidores dos clientes e das ETC. Foi gerado um banco de dados das medições entre 01 a 05 de dezembro de 2018, com medições sempre diárias, relacionando as ETC com os oito clientes consumidores de gás natural. Logo após, é aplicada a reconciliação de dados de forma que, a aglutinação e posterior 
comparação monetária entre as diferenças de medições reconciliadas e não reconciliadas sejam feitas de forma sucinta. Como instrumento para realização desta pesquisa foi utilizado o software Microsoft Excel.

\section{RESULTADOS E DISCUSSÃO}

Como os medidores de vazão estão com certificados de calibração dentro dos prazos e incertezas de medição estabelecidas pelos órgãos reguladores, parte-se do pressuposto que não há erros grosseiros no banco de dados em pauta. 2018:

Segue abaixo as medições realizadas entre os dias 01 a 05 de dezembro de

Incertezas expandidas dos medidores de vazão volumétrica:

\begin{tabular}{|c|c|}
\hline PONTOS DE MEDIÇÃO & INCERTEZA EXPANDIDA (\%) \\
\hline ETC & 0,62 \\
\hline CLIENTE A & 0,66 \\
\hline CLIENTE B & 0,70 \\
\hline CLIENTE C & 0,59 \\
\hline CLIENTE D & 0,54 \\
\hline CLIENTE E & 0,50 \\
\hline CLIENTE F & 0,58 \\
\hline CLIENTE G & 0,68 \\
\hline
\end{tabular}

\section{Dados Não Reconciliados:}

Figura 1 - Gráfico ETC x Somatório Clientes(m³/dia)

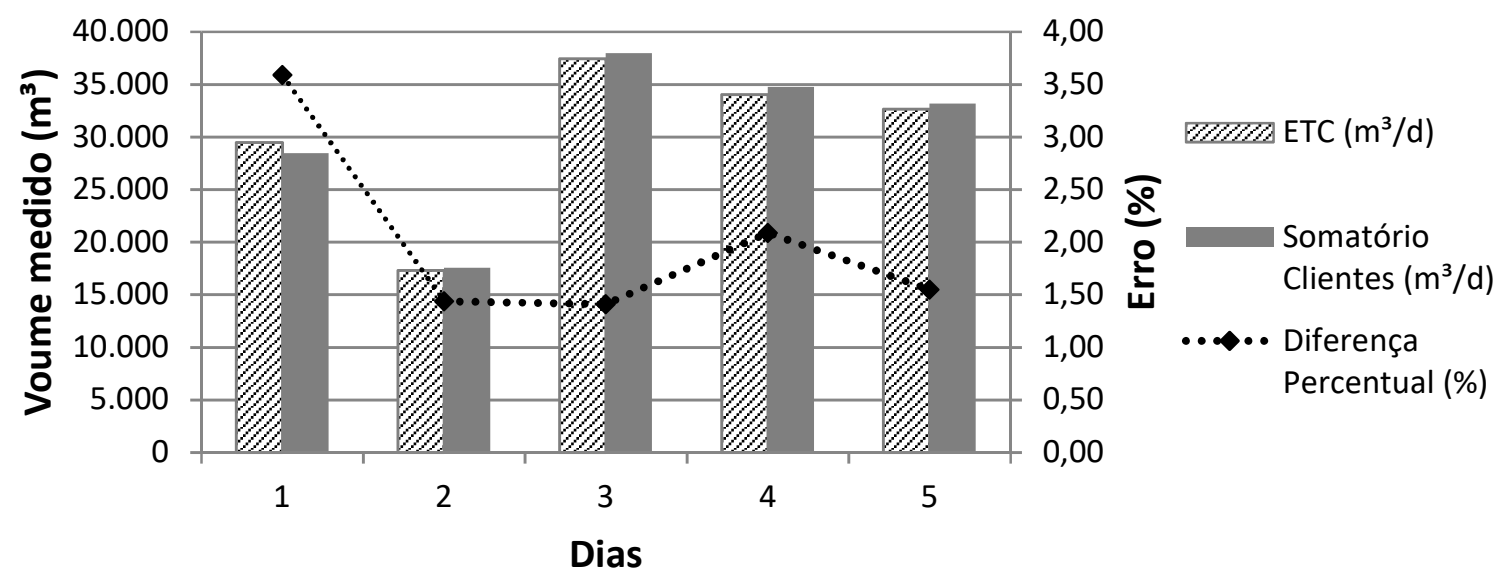


V SIINTEC $\begin{aligned} & \text { INTERNATIONAL SYMPOSIUM ON INNOVATION AND TECHNOLLGY } \\ & \text { SIMPOSIO INTERNACIONAL DE INOVACAO ETECNOLOGIA }\end{aligned}$

\begin{tabular}{l|l} 
CIRCULAR ECONOMY & ECONOMIA CIRCULAR
\end{tabular}

Figura 2 - Medição de vazão por Cliente
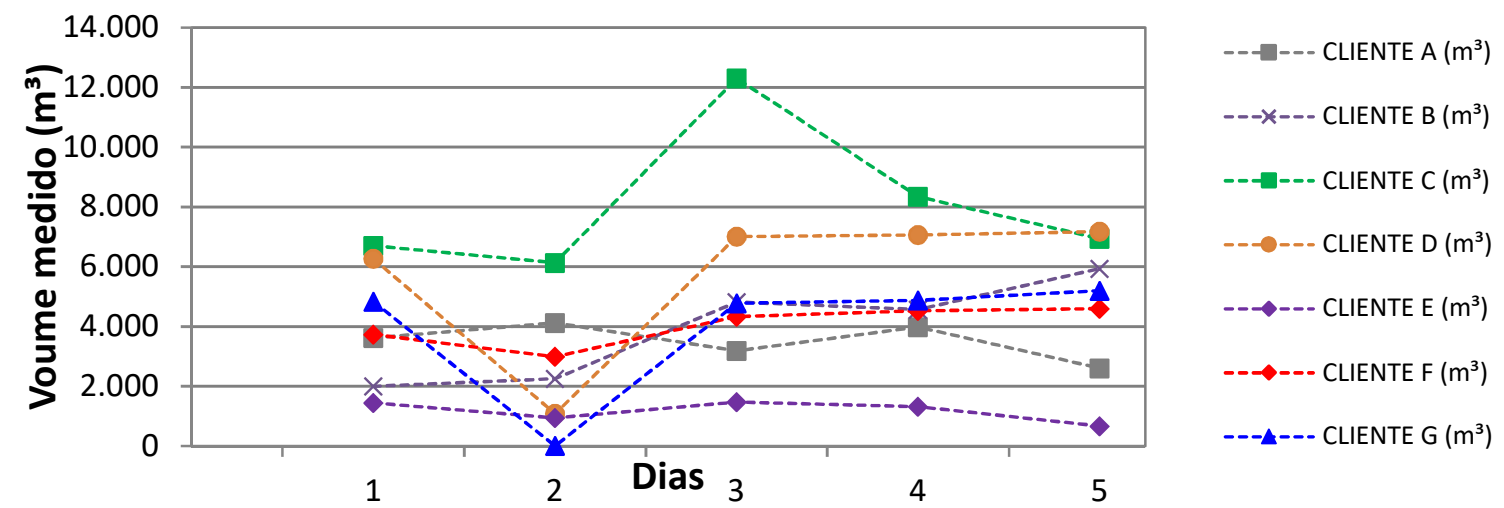

\section{Dados Reconciliados:}

Figura 3 - ETC x Somatório Clientes

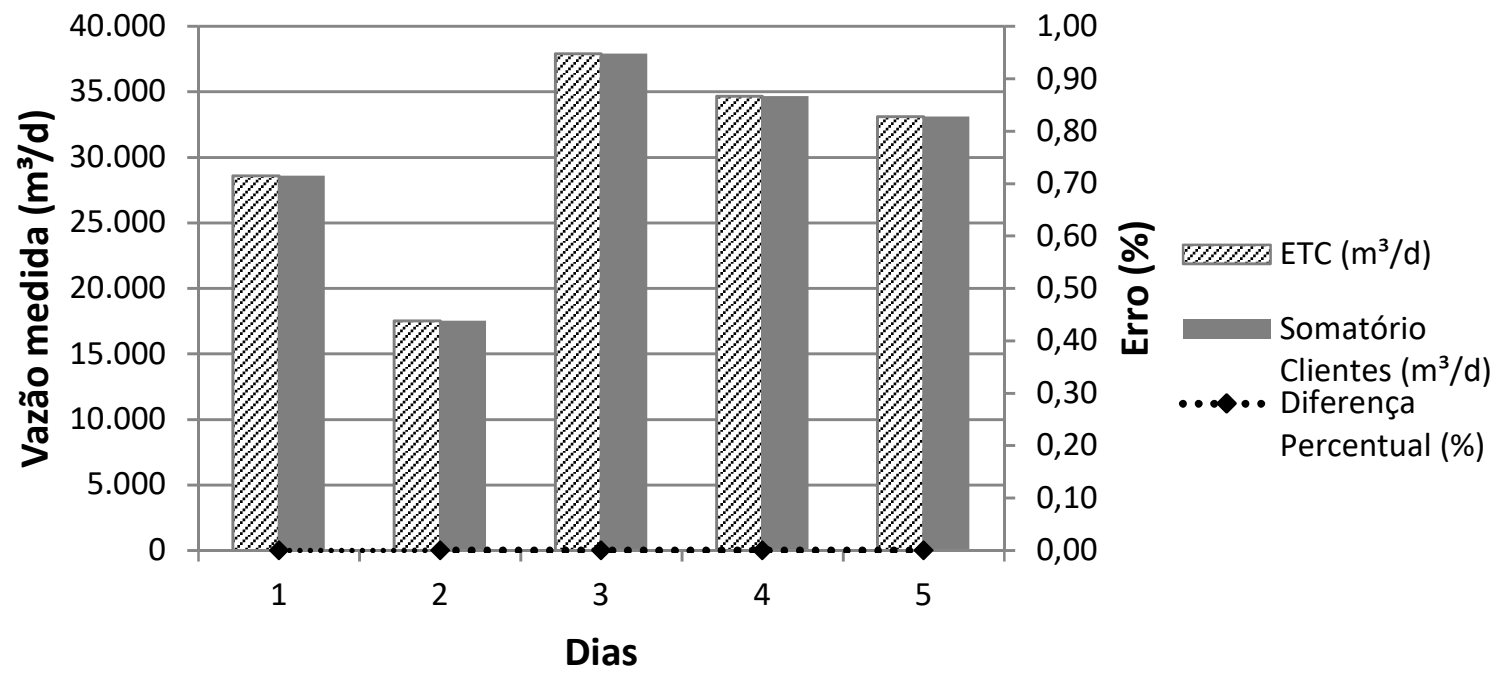

Figura 4 - Medição por Cliente ( $\left.\mathrm{m}^{3} / \mathrm{dia}\right)$

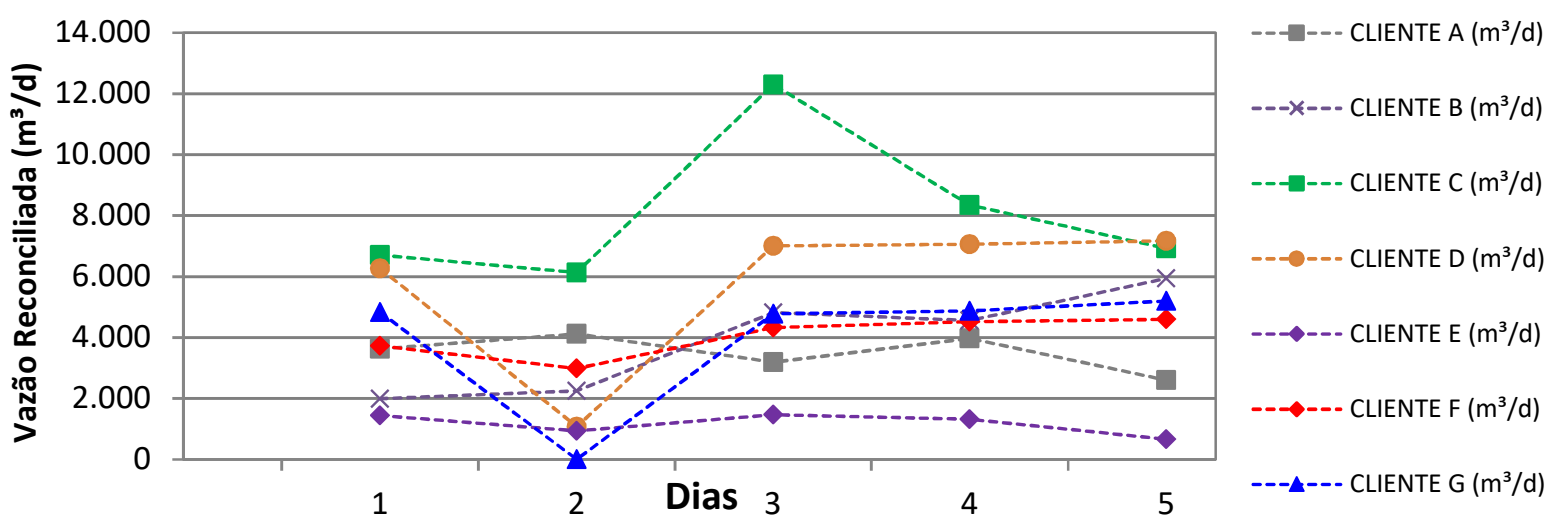


Figura 5 - Diferença percentual entre dados medidos e reconciliados (\%)

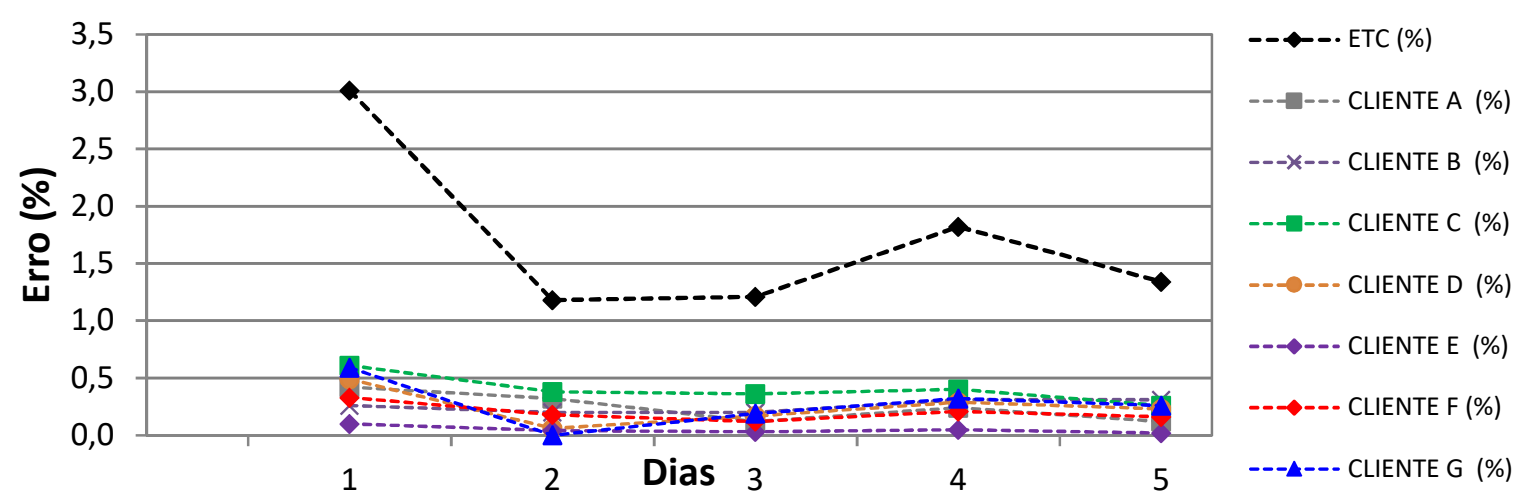

Tabela 2 - Projeção monetária anual $(\mathrm{R} \$)$ da diferença entre dados reconciliados e medidos (sem impostos) ${ }^{[10]}$

\begin{tabular}{|c|c|c|c|c|c|c|c|}
\hline ETC & CLIENTE A & CLIENTE B & CLIENTE C & CLIENTE D & CLIENTE E & CLIENTE F & CLIENTE G \\
\hline $\mathrm{R} \$$ & $\mathrm{R} \$$ & $\mathrm{R} \$$ & $\mathrm{R} \$$ & $\mathrm{R} \$$ & $\mathrm{R} \$$ & $\mathrm{R} \$$ & $\mathrm{R} \$$ \\
\hline $105.585,86$ & $-1.961,22$ & $-5.533,59$ & $-10.409,45$ & $-2.518,57$ & $-24,83$ & $-1.925,58$ & $-1.242,50$ \\
\hline
\end{tabular}

As tabelas e figuras acima mostram de forma comparativa os dados medidos e reconciliados das medições de vazão, tanto ao se comparar a medição da Estação de Transferência de Custódia com o somatório dos 7 clientes, quanto na comparação por cliente.

Na figura 1 (antes da reconciliação), nota-se a pequena diferença no balanço de medição, ou seja, na diferença de medição entre o somatório de clientes e ETC. Consequentemente, essa pequena diferença no balanço global (ETC x Clientes) revela que as medições de gás natural estão dentro da margem de incerteza e tratam-se de dados confiáveis.

Ao se observar a figura 5, nota-se que as diferenças entre dados reconciliados e medidos é pequena. Isso fica claro, comparando-se as medições por clientes. É mais um motivo comprobatório da confiabilidade nas medições.

Por conseguinte, avaliando-se a diferença pelo âmbito de faturamento (tabela 2), segue a mesma lógica descrita para as medições, ou seja, a diferença de faturamento entre dados medidos e reconciliados é pequena. Um ponto importante para análise são as diferenças entre as medições reconciliadas e não reconciliadas na ETC. Como as diferenças são maiores, consequentemente apresentarão discrepâncias mais acentuadas relacionadas ao faturamento.

\section{CONCLUSÃO}

Foi realizada uma avaliação para medição de gás natural na concessionaria de distribuição de gás natural da Bahia, tomando-se como modelo um "recorte" de parte da malha medição, comparando-se as medições entre as ETC (Estações de Transferência de Custódia) e os pontos de consumo para os diversos clientes dessa 
concessionária, aplicando a técnica de Reconciliação de Dados, verificando-se a relação entre consumo e fornecimento.

A aplicação da técnica de reconciliação de dados provou ser uma ferramenta bastante útil de avaliação do balanço volumétrico da malha de gasodutos.

Os resultados mostraram o pequeno distanciamento entre o balanço de gás medido e reconciliado, com consequente pequena diferença de faturamento. Isso mostra que a medição de gás natural na concessionária em pauta é confiável.

\section{REFERÊNCIAS}

${ }^{1}$ BARRETO, G. O. Reconciliação de dados do balanço de massa no gasoduto Urucu-Manaus. Dissertação (Programa de Pós-Graduação em Metrologia) Pontifícia Universidade Católica do Rio de Janeiro. 2015.

2 BAI, S., MCLEAN, D. D., THINBAULT, J., 2007, "Impact of Model Structure on the Performance of Dynamic Data Reconciliation", Computers and Chemical Engineering, v. 31, pp. 127-135.

${ }^{3}$ CROWE, C. M, 1986. Reconciliation of process flow rates by Matrix Projection. AIChE journal, v.32, nำ4, p. 616-623.

4 KNOPF, CARL.F, 2012, Modeling, Analysis and Otimization of Process and Energy System. 1 ed. New Jersey, Published by John Wiley \& Sons.

${ }^{5}$ MARTINS, MARCIO et.al., 2009, "Utilização de questionários na estimativa da incerteza em reconciliação de dados do balanço hídrico", Uberlândia, MG, Brasil.

6 NARASIMHAN, S., JORDACHE, C., 2000, Data reconciliation and gross error detection: an intelligent use of process data. 1 ed. Houston, Gulf Publishing Company.

${ }^{7}$ OLIVEIRA JR, A. M., 2006, Estimação de Parâmetros em Modelos de Processo Usando Dados Industriais e Técnicas de Reconciliação de Dados, Tese de M.Sc., COPPE/UFRJ, Rio de Janeiro, RJ, Brasil.

8 PRATA, D. M., 2005, Reconciliação de Dados em um Reator de Polimerização, Tese de M.Sc., COPPE/UFRJ, Rio de Janeiro, RJ, Brasil.

${ }_{9}^{9}$ RIBEIRO, Marco Antônio. Curso de medição de petróleo e gás natural. 3.ed., 2003.355p.

${ }^{10}$ http://www.bahiagas.com.br/gas-natural/tabela-tarifaria. Acesso em: 10/08/2019. 